
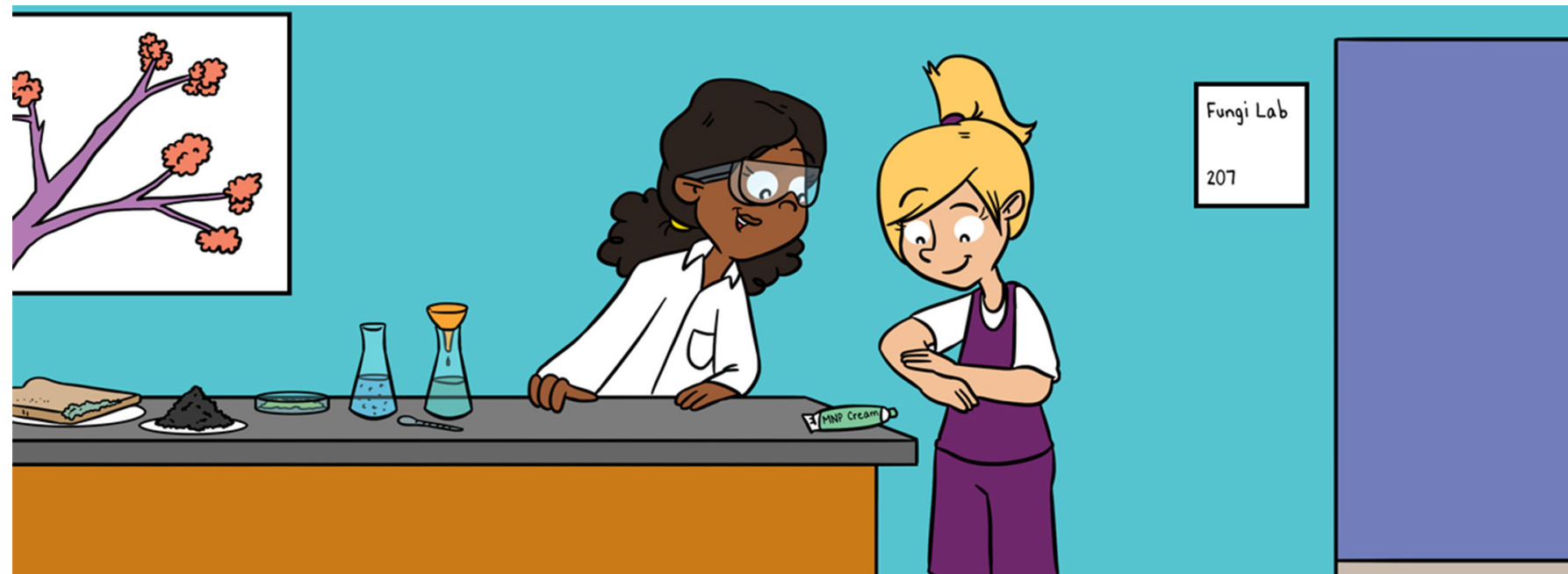

\title{
A MOLDY WAY TO FIGHT INFECTIONS-THROUGH METAL NANOPARTICLES
}

\author{
Jessica Berry ${ }^{1}$, Cristiane A. Ottoni ${ }^{2}$ and Marta Filipa Simões ${ }^{3,4^{*}}$ \\ ${ }^{1}$ Royal Hallamshire Hospital, Sheffield Teaching Hospitals National Health Service (NHS) Foundation Trust, Sheffield, \\ United Kingdom \\ ${ }^{2}$ Bioscience Institute, Universidade Estadual Paulista Júlio de Mesquita Filho (UNESP), São Vicente, Brazil \\ ${ }^{3}$ State Key Laboratory of Lunar and Planetary Sciences, Macau University of Science and Technology, Macau, China \\ ${ }^{4}$ China National Space Administration (CNSA), Macau Center for Space Exploration and Science, Macau, China
}

YOUNG REVIEWER:

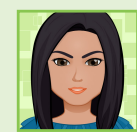

MRITTIKA

AGE: 13
Many microbes, which are tiny organisms capable of causing disease, have developed resistance to the common drugs used against them. Alternative drugs are being developed to fight these resistant microbes. One of these alternatives is the use of metal nanoparticles, which are extremely tiny particles of metals like silver, gold, or copper. Metal nanoparticles can be used directly against microbes or as complements to traditional therapies. They can also be used for many other applications in various industries. Filamentous fungi, commonly called molds, can be used to produce alternative ways to fight microbial infections. Here, as an example, we explain the production of silver nanoparticles by filamentous fungi, and how these nanoparticles could be used. 


\section{METABOLITES}

Chemical substances released during the active metabolism of a microbe, in our case fungi. Some types of metabolites are drugs, like antibiotics, as well as pigments used for food coloring.

\section{ANTIBIOTIC}

Substance used to treat or prevent infections caused by bacteria.

\section{ANTIMICROBIAL} RESISTANCE

Capacity of some microbes to resist to the exposure of drugs intended to kill them, and to continue to grow.

\section{METAL}

NANOPARTICLES

Very small particles, with sizes ranging from 1 to $100 \mathrm{~nm}$, of metal. Much smaller than most microbes.

\section{FILAMENTOUS}

FUNGI

Fungi that form filaments called mycelia.

\section{INTRODUCTION}

Fungi are a diverse group of organisms and they are everywhere. There are many known species, but we have not discovered them all yet. In fact, we have only managed to describe $8-10 \%$ of all fungal species. This leaves much to be explored, studied, and understood [1]. Fungi can have different appearances, sizes, and colors, depending on where they grow. When observed under a microscope, we can see their structures in detail like in Figure 1. The amount of detail depends on the type of microscopy that we use (see Box 1).

You can see an increase in detail from stereomicroscopy (Figure 1C), to optical microscopy (Figure 1D), and then to scanning electron microscopy (SEM) (Figure 1E).

Fungi have adapted to each place they inhabit. One adaptation is their ability to create several chemical substances, called metabolites. Fungi use their metabolites as weapons against bacteria or even other fungi. Humans can also make use of some of the metabolites as antibiotics to fight infections caused by bacteria, or even use them to fight cancer [1].

\section{WHY DO WE NEED FUNGI TO HELP US FIGHT INFECTIONS?}

Drugs are becoming less and less effective against pathogenic microbes. This is a worrying worldwide problem, leading to diseases that are not easy to treat and microbes that are not easy to kill. When exposed to medicines, microbes can adapt. Then, if they develop the capacity to survive, they turn into superbugs, with the capacity of antimicrobial resistance. We need urgent alternatives to our current drugs. Metal nanoparticles (MNPs) are part of the ongoing wave of exploration of such alternatives. These are extremely tiny particles of metals that can be made of silver, gold, or copper.

Scientists have discovered that fungi can be used to produce MNPs. Production of MNPs by fungi is sustainable, meaning that large amounts can be produced faster and cheaper than other production methods, and it does not produce chemical hazardous waste. The most useful types of fungi for production of MNPs are molds. Molds, also known as filamentous fungi, are different from mushrooms. They form filaments or mycelia (Figure $1 \mathrm{C}$ ). Molds are very sturdy organisms and can accumulate metals from the surrounding environment. This makes them ideal producers of MNPs. 
Figure 1

Different views of one strain of Penicillium sp., with increasing detail.

(A,B) Petri dishes

(diameter $=90 \mathrm{~mm}$ )

with colonies as seen with the naked eye,

from the front (A) and

the back (B). (C)

Stereomicroscopy

analysis of fungal

mycelia or filaments

( $\times 10$ magnification,

scale bar: $75 \mu \mathrm{m})$. (D)

Optical microscopy

(400x magnification,

scale bar: $20 \mu \mathrm{m})$. (E)

Scanning electron

microscopy image of

fungal spores $(8,000 x$

magnification, scale

bar: $5 \mu \mathrm{m}$ ).

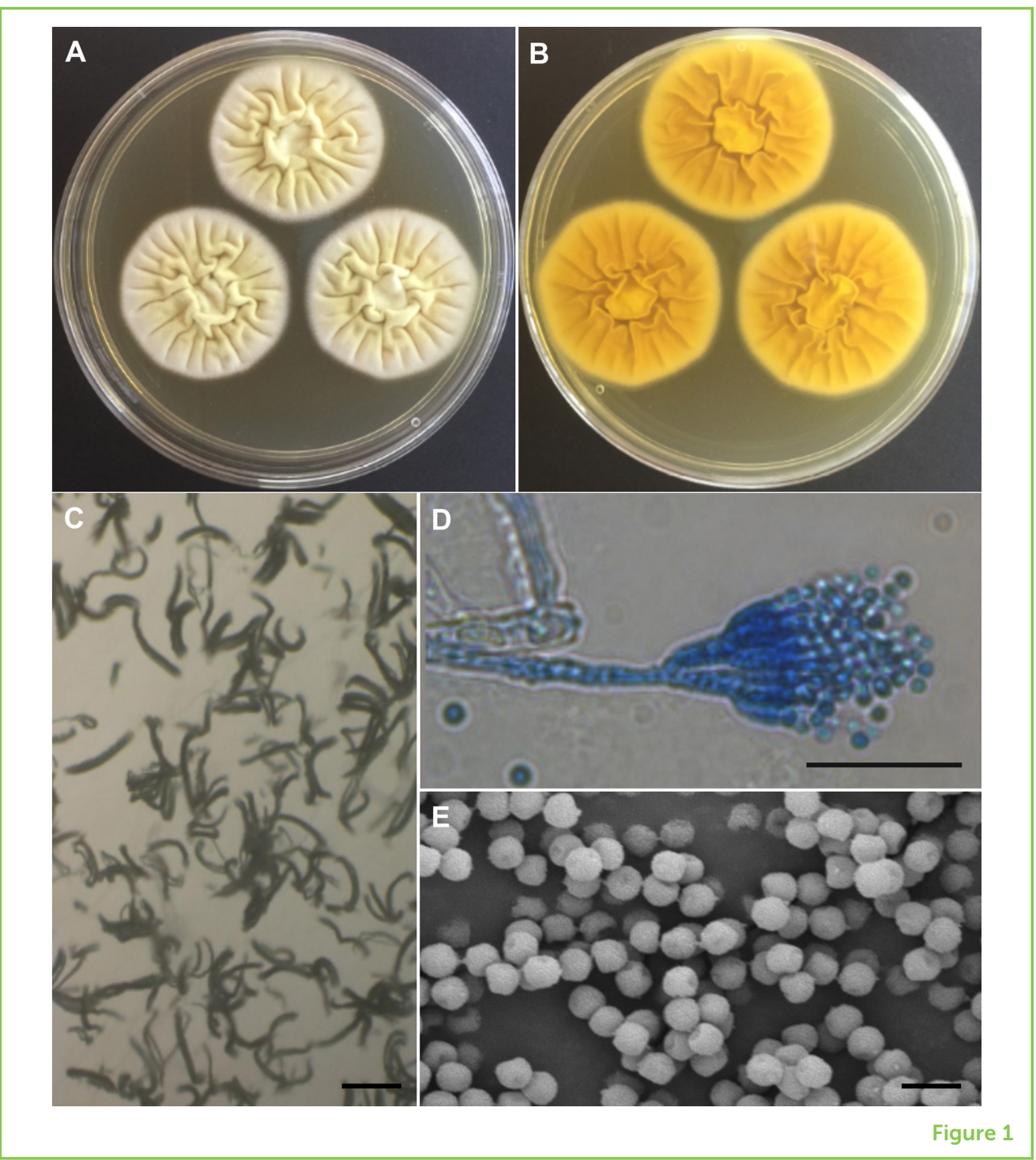

HOW ARE METAL NANOPARTICLES PRODUCED?

MNPs can be made of different metals, including copper, cobalt, palladium, selenium, platinum, and lead. But, the most researched and used MNPs are silver nanoparticles (AgNPs) and gold nanoparticles (AuNPs). AgNPs and AuNPs have been shown to have antimicrobial activity, which means they can kill or prevent the growth of many microbes. This activity is not completely understood. However, we know that some metal ions released from the MNPs (AgNP, Figure 2E) can inhibit bacterial and fungal growth [2]. We also know that both AgNP and AuNP work by damaging the cell membranes of microbes. The antimicrobial activity of the nanoparticles can be affected by their surface, composition, and amount. MNPs also have different effects depending on the type of microbes they are used against [3].

MNPs can be made by several different processes. These processes can be physical, chemical, or biological [4]. Chemical synthesis uses potentially hazardous chemicals and generates toxic waste products. 


\section{Box 1 | Microscopy types}

There are several different microscopes that vary in the type of images obtained, size, price, and complexity. More complex microscopes will show higher amounts of detail, but will be bigger, more complex to use, and more expensive. You can see an increase in detail from stereomicroscopy, to optical microscopy, and then to electron microscopy. Electron microscopy can be scanning electron microscopy (SEM) or transmission electron microscopy (TEM). See Figure 1 for an example of the levels of detail that can be seen with different kinds of microscopes.

\begin{tabular}{ll}
\hline Microscope & Description \\
\hline Stereomicroscope & Also called dissecting microscope, uses \\
& regular light to form images, provides a low \\
magnification of up to 300 times. & Also uses regular light to form images but \\
Optical microscope & times. It is the most common type of \\
& microscope used. \\
Scanning electron & images from samples analyzed in vacuum. \\
microscope (SEM) & Can produce a magnification of 1-3 million \\
& times. \\
Transmission electron & Also uses electrons instead of light, but \\
microscope (TEM) & forms images from samples prepared in \\
slides or grids (like slices of bacteria) and \\
with some degree of transparency, \\
presenting increased details. Offers much \\
higher magnifications up to 50 million times.
\end{tabular}

BIOLOGICAL

SYNTHESIS

Production or formation of compounds from simpler elements, mediated by living beings or organic substances from organisms. In our case the biological synthesis of MNPs is promoted by fungi.

\section{SUPERNATANT}

Remaining liquid solution, after removal of all solid substances.
These waste products, if released into the environment, will affect the ecosystem. Physical synthesis uses mechanical methods (like crushing) to change large-sized pieces of metal into nano-scale sized particles [4]. Biological synthesis of MNPs, which means their formation using living organisms (in this case fungi), is often the preferred method. It is considered an eco-friendlier technique when compared to others. Its resulting MNPs are more compatible with living tissues and less toxic for medical analyses; and, many microbes, including fungi, can serve as eco-friendly nanofactories of MNPs [3].

Biological synthesis of MNPs using fungi is a relatively simple process. This is because fungi secrete large amounts of metabolites that can be used to produce MNPs. For example, to produce AgNPs from filamentous fungi (Figure 2), the fungi must first be grown in an appropriate growth medium (one where the fungus grows better and which might be different for each fungal species), which is like a broth. After they have grown for a while, the fungal cells are removed from the broth and put in water, where the cells release metabolites. The fungal cells are then removed and the water containing the metabolites is kept. This solution, free from fungi, is called the supernatant. The supernatant is used to form MNPs through a biological process that happens when certain chemicals are added to the supernatant. Formation of MNPs is noticeable by the change of color from yellow to brown, but it can then be further analyzed by 
Figure 2

(A) Small pieces are cut from fully grown fungal colonies (in Petri dishes) and transferred into liquid broth where further growth will occur (4-5 days). (B) The fungal cells are removed from the broth and placed in sterile water, where they release metabolites. (C) After 1 day, the fungal cells are removed by filtration and the supernatant is kept. (D) A chemical is then added to the supernatant and left for some time (varies from hours to days) until MNPs are produced. Their production is detected by color change or microscopy. (E) Here you see a TEM image of AgNPs synthesized by a strain of Penicillium citrinum.
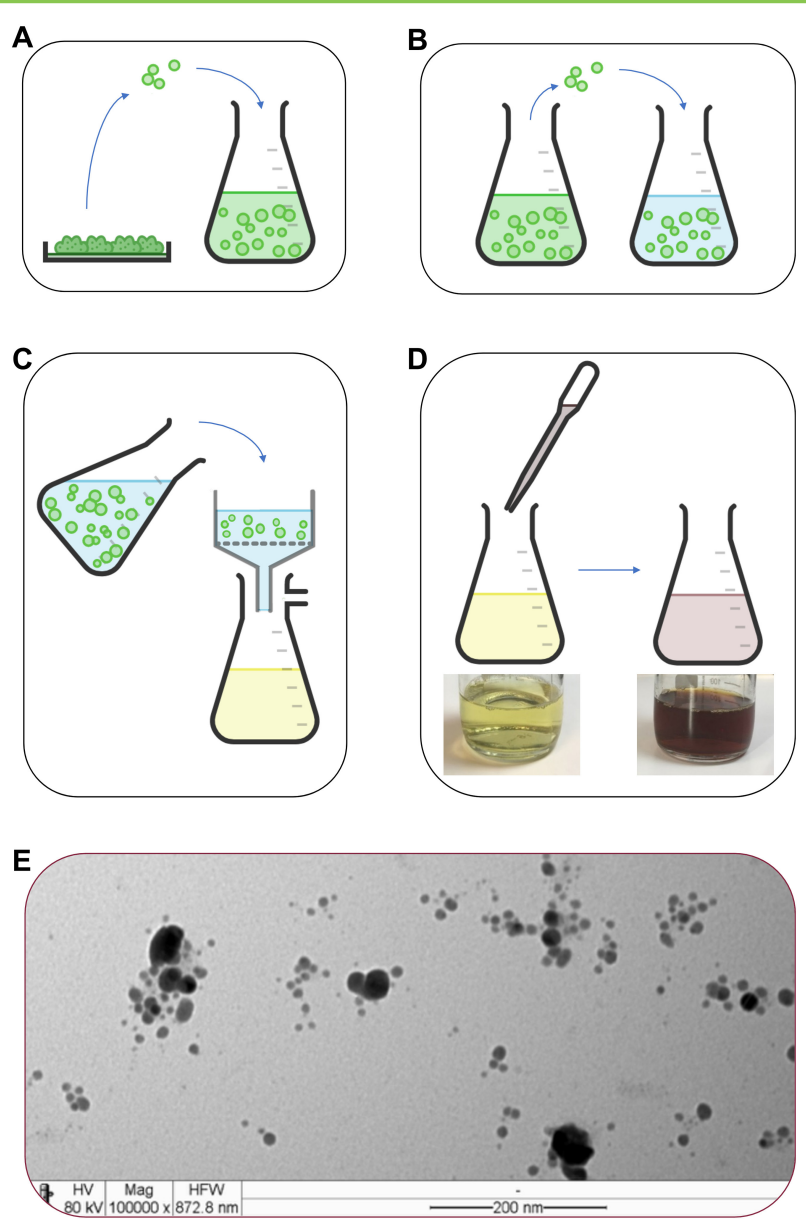

Figure 2

several complex techniques, like electron microscopy, to make sure MNPs have been produced (Figure 2E) [3]. For the formation of AgNPs, the chemical added to the supernatant is silver nitrate. The yellow supernatant obtained from the fungus Penicillium sp. will turn brown after 4 days of incubation with silver nitrate, resulting in AgNPs.

\section{OTHER USES OF MNPS}

As mentioned, MNPs can be used to fight infections caused by bacteria, fungi, or viruses. But they can also be used to detect infections as part of medical tests, or in food packaging, to help prevent microbes from contaminating the food. They can be added to the materials used to make medical equipment, preventing microbes from attaching to this equipment. This could help in preventing infections, for example during surgeries. MNPs in the form of ointments, topical creams, or solutions can be applied directly to wounds. MNPs can be mixed with other materials or chemicals, as a part of other antimicrobial drugs, or can even be used as a vehicle to deliver other compounds, by carrying them to specific target cells. MNPs can also have anti-cancer activity, they can be used as an ingredient in cosmetics, or used in batteries and textiles. They have applications 
in industries very different from the medical and pharmaceutical sectors, including agriculture, food industry, in energy and automobile industries, and in many biotechnology fields $[2,4]$.

\section{CONCLUSION}

Filamentous fungi (or mold) are easy to manipulated in the laboratory, simple to grow, able to form large amounts of cells, at relatively low costs. This makes them ideal organisms for biological processes, such as the production of MNPs.

As you have learned, there are many uses of MNPs in the fight against a wide range of infections. But there is much yet to explore. With the discovery of new filamentous fungal species, we might be able to develop better and more efficient biological processes to produce MNPs with higher antimicrobial activity against infectious microbes. Such MNPs might help in the fight against superbugs.

\section{REFERENCES}

1. Simões, M. F., Pereira, L., Santos, C., and Lima, N. 2013. Polyphasic identification and preservation of fungal diversity: Concepts and applications, in: Management of Microbial Resources in the Environment, eds A. Malik, E. Grohmann, and M. Alves (Dordrecht: Springer). p. 91-117. doi: 10.1007/978-94-007-5931-2_5

2. Mandal, D., Bolander, M. E., Mukhopadhyay, D., Sarkar, G., and Mukherjee, P. 2006. The use of microorganisms for the formation of metal nanoparticles and their application. Appl. Microbiol. Biotechnol. 69:485-92. doi: 10.1007/s00253-005-0179-3

3. Ottoni, C. A., Simões, M. F., Fernandes, S., Dos Santos, J. G., Da Silva, E. S., de Souza, R. F. B., et al. 2017. Screening of filamentous fungi for antimicrobial silver nanoparticles synthesis. AMB Express 7:31. doi: 10.1186/s13568-017-0332-2

4. Simões, M. F., Ottoni, C. A., and Antunes, A. 2020. Biogenic metal nanoparticles: a new approach to detect life on Mars? Life 10:28. doi: 10.3390/life10030028

SUBMITTED: 31 March 2020; ACCEPTED: 12 October 2020;

PUBLISHED ONLINE: 06 November 2020.

EDITED BY: Michel Goldman, Institute for Interdisciplinary Innovation in healthcare (I3h), Belgium

CITATION: Berry J, Ottoni CA and Simões MF (2020) A Moldy Way to Fight Infections-Through Metal Nanoparticles. Front. Young Minds 8:547529. doi: 10. 3389/frym.2020.547529

CONFLICT OF INTEREST: The authors declare that the research was conducted in the absence of any commercial or financial relationships that could be construed as a potential conflict of interest. 


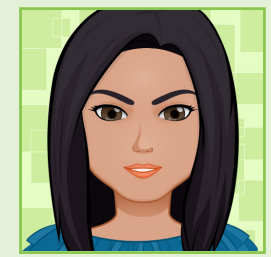

COPYRIGHT (c) 2020 Berry, Ottoni and Simões. This is an open-access article distributed under the terms of the Creative Commons Attribution License (CC BY). The use, distribution or reproduction in other forums is permitted, provided the original author(s) and the copyright owner(s) are credited and that the original publication in this journal is cited, in accordance with accepted academic practice. No use, distribution or reproduction is permitted which does not comply with these terms.

\section{YOUNG REVIEWER}

\section{MRITTIKA, AGE: 13}

Mrittika loves hanging out with her friends and family. Her interests include: playing the viola and ukulele, dancing, poetry, singing, reading, and calligraphy. Math, Social Studies, and Music are among her favorite subjects. Her favorite sports are volleyball, karate, and running. Mrittika's favorite accomplishments are becoming a senior editor on her yearbook editing team, and getting onto her school's show choir. She is also her school's geography bee champion and aspires to be a more open-minded and knowledgeable person.

\section{AUTHORS}

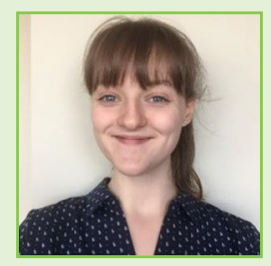

\section{JESSICA BERRY}

I am a Biology graduate from Edge Hill University (EHU), England, where I took a particular interest in both microbiology and laboratory research. I carried out a summer research internship on fetal growth restriction in mice, in which I gained histological and laboratory skills. My research for my final year project focused on the antimicrobial potential of metallic nanoparticles, specifically those synthesized by fungi, to combat harmful microbial diseases, such as tuberculosis. I currently work within the National Health Service (NHS), as part of the Histopathology team, where I process and prepare diseased tissue for diagnosis by trained pathologists.

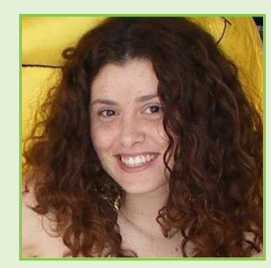

\section{CRISTIANE A. OTTONI}

I am currently a lecturer at the Universidade Estadual Paulista Júlio de Mesquita Filho (UNESP), São Paulo, Brazil. Most of my work combines chemistry, biotechnology and microbiology. I have been developing my research in applied nanotechnology, where I focus on studying nanoparticle synthesis and characteristics, as well as their application in several diverse industries and their environmental impact. I also study several other biotechnological processes and different designs and applications of microbial fuel cells.

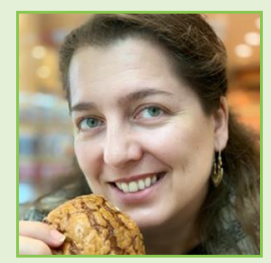

\section{MARTA FILIPA SIMÕES}

I work in astrobiology at the State Key Laboratory of Lunar and Planetary Science (SKLPlanets), at Macau University of Science and Technology (MUST), China. I am a microbiologist who has worked with a myriad of microorganisms (mycobacteria, environmental and clinical bacteria, mycobacteriophages, and filamentous fungi) in several different countries (UK, Saudi Arabia, and Portugal). I study mostly fungal ecology and diversity in environments that are similar to outer-space conditions, I search for new filamentous fungal species or species with new and useful capabilities, and I look into fungal growth to control and stop potential contaminations. *msimoes@must.edu.mo 